\title{
Aves de rapina (Cathartiformes, Accipitriformes, Strigiformes e Falconiformes) na Reserva Biológica das Perobas, Paraná, Brasil, e seu entorno
}

\author{
Willian Menq ${ }^{1 *}$ \\ Rosilene Luciana Delariva ${ }^{2}$ \\ ${ }^{1}$ Aves de Rapina Brasil \\ Rua Marino Paulichi, 960, CEP 87060-330, Maringá - PR, Brasil \\ ${ }^{2}$ Universidade Estadual do Oeste do Paraná, Cascavel - PR, Brasil \\ * Autor para correspondência \\ willianmenq@gmail.com
}

Submetido em 28/04/2015

Aceito para publicação em 30/09/2015

\section{Resumo}

Nas últimas décadas, o noroeste do Paraná sofreu uma drástica supressão e fragmentação de sua vegetação primária, restando atualmente pequenos e esparsos fragmentos florestais. Nessa perspectiva, este estudo teve por objetivos inventariar e analisar a assembleia de aves de rapina (Cathartiformes, Accipitriformes, Strigiformes e Falconiformes) na Reserva Biológica das Perobas, uma unidade de conservação de 8.716 ha, a qual representa um dos últimos remanescentes com extensão significativa de Floresta Estacional Semidecidual no Paraná. Foi utilizado o método de contagem por pontos fixos, bem como pontos de escuta e playback, para amostrar as espécies de aves de rapina da reserva, entre junho de 2009 a janeiro de 2012, com esforço amostral total de 210 h. Ao todo, foram levantadas 25 espécies de rapinantes, valor que corresponde a $37 \%$ da riqueza conhecida para o Paraná. Entre os registros, três espécies merecem ser destacadas, Spizaetus melanoleucus, Asio flammeus e Strix virgata, listadas no atual Livro Vermelho da Fauna Ameaçada do Paraná e pouco documentadas na região. Cerca de $70 \%$ das espécies observadas apresentam hábitos associados a ambientes florestais, enquanto que $30 \%$ estão associadas a áreas abertas, como as pastagens e as terras de cultivo no entorno da floresta. Esses resultados reafirmam a importância dessa reserva para a conservação e manutenção de muitas espécies de aves, principalmente as raras e regionalmente ameaçadas de extinção.

Palavras-chave: Asio flammeus; Avifauna; Floresta Estacional Semidecidual; Spizaetus melanoleucus; Strix virgata

\section{Abstract}

Birds of prey (Cathartiformes, Accipitriformes, Strigiformes e Falconiformes) in the Perobas Biological Reserve, Parana, Brazil, and its surroundings. In recent decades, the northwest of the state of Paraná, Brazil, has undergone a dramatic suppression and fragmentation of its primary vegetation, currently there remains small and sparse forest fragments. From this perspective, this study aimed to do an inventory of and analyze the assembly of birds of prey (Cathartiformes, Accipitriformes, Strigiformes, and Falconiformes) in the Perobas Biological Reserve, a conservation area of 8,716 ha, which represents one of the last remnants with significant extent of semideciduous seasonal forest in Paraná. The counting method by fixed sites, as well as 
hearing sites and playback, was used to sample the bird of prey species in the reserve, from June 2009 to January 2012 , with a total sampling effort of $210 \mathrm{~h}$. Altogether 25 raptor species were surveyed, a value corresponding to $37 \%$ of the species richness known for Paraná. Among the records, 3 species are worth highlighting, Spizaetus melanoleucus, Asio flammeus, and Strix virgata, listed in the current Red Book of Endangered Fauna in Paraná and poorly documented in the region. About $70 \%$ of the observed species have habits associated with forest environments, while $30 \%$ are associated with open areas, such as pastures and farmland surrounding the forest. These results reaffirm the importance of this reserve for the conservation and preservation of many bird species, especially those rare and regionally endangered.

Key words: Asio flammeus; Birds; Semideciduous Forest; Spizaetus melanoleucus; Strix virgata

\section{Introdução}

O noroeste do Paraná passou por uma rápida transformação a partir da década de 1970, especialmente devido ao avanço da fronteira de colonização, bem como dos processos de mecanização e plantio de culturas anuais (CAMPOS, 1998). Nesse cenário, essa região foi descaracterizada por atividades antrópicas através da supressão e fragmentação da vegetação primária, restando atualmente menos de $10 \%$ de sua cobertura original (STRAUBE; URBEN-FILHO, 2005). Originalmente recoberta pela Floresta Estacional Semidecidual, atualmente essa região é representada por áreas agrícolas e pastagens, além de pequenos e esparsos fragmentos florestais que raramente excedem 300 ha (STRAUBE et al., 1996; ANJOS, 1998; LOURESRIBEIRO; ANJOS, 2006).

A Reserva Biológica das Perobas (RBP), localizada nos municípios de Tuneiras do Oeste e Cianorte, com seus 8.716 ha, é o maior remanescente de Floresta Estacional Semidecidual da região norte e noroeste do estado do Paraná, sendo um refúgio de fauna e flora importante da região (ICMBIO, 2012). A área abriga uma grande variedade de espécies, com destaque para aves raras e ameaçadas nas listas regionais e nacional, como o jaó (Crypturellus undulatus), macuco (Tinamus solitarius), araçari-de-bico-branco (Pteroglossus aracari), pavó (Pyroderus scutatus), dentre outras (ICMBIO, 2012). Entretanto, a avifauna da reserva ainda é pouco conhecida. Embora já tenham sido catalogadas 160 espécies de aves, em função de suas dimensões e características vegetacionais, estima-se que pelo menos 300 espécies ocorram na área (ANJOS; MENQ, 2014).

Dentre as espécies de aves catalogadas na reserva, o grupo das aves de rapina, compreendidas pelas ordens Accipitriformes, Falconiformes, Cathartiformes e Strigiformes, é um dos menos conhecidos e mais subamostrados. A carência de registros de muitas das espécies desse grupo se deve principalmente pelos seus hábitos inconspícuos e as suas baixas densidades populacionais (OLMOS et al., 2006; GRANZINOLLI; MOTTA-JUNIOR, 2008).

No Paraná são conhecidas 68 espécies de rapinantes (SCHERER-NETO et al., 2011), sendo que dessas, pelo menos 41 ocorrem na região noroeste (STRAUBE et al., 1996; STRAUBE; URBEN-FILHO, 2005). Entretanto, a maioria possui escassos registros recentes, especialmente as espécies de hábitos florestais, o que dificulta a categorização de cada uma em relação ao status na natureza (SANTOS et al., 2009).

Nesse sentido, esse estudo teve por objetivos inventariar as aves de rapina (Cathartiformes, Accipitriformes, Strigiformes e Falconiformes) na Reserva Biológica das Perobas, bem como sua área de entorno. Essas informações são relevantes contribuições no conhecimento da ocorrência desse grupo de aves, bem como do uso dessa unidade de conservação e seu papel como refúgio biológico no noroeste do Paraná.

\section{Material e Métodos}

\section{Área de estudo}

O estudo foi realizado na Reserva Biológica das Perobas (RBP) - 23⒋ $47^{\prime}$ e $23^{\circ} 54^{\prime} \mathrm{S}-52^{\circ} 41^{\prime}$ e $52^{\circ} 50^{\prime} \mathrm{W}$, Unidade de Conservação (UC) federal de proteção integral, localizada nos município de Tuneiras do Oeste e Cianorte, noroeste do estado do Paraná (ICMBIO, 2012). Com uma área de 8.716 ha, é o maior remanescente 
florestal da região, situado em zona de contato entre a Floresta Estacional Semidecidual e a Floresta Ombrófila Mista (ICMBIO, 2012). De acordo com os critérios de Koeppen, a área apresenta um clima regional típico $\mathrm{Cfa}$ (tropical-subtropical) no qual apresenta chuvas bem distribuídas ao longo do ano e verões rigorosos (PEEL et al., 2007).

\section{Procedimentos de campo}

As amostragens foram realizadas entre junho de 2009 e janeiro de 2012, em cinco pontos amostrais distribuídos em diferentes áreas da RBP, além do uso de uma trilha, de $8.300 \mathrm{~m}$, que corta o interior da UC de leste a oeste (Figura 1), de modo a se obter uma melhor representatividade das variações florísticas da reserva, como as áreas em regeneração, floresta primária, secundária e bordas. Revisões de literatura, amostragens esporádicas externas aos pontos amostrais bem como informações de terceiros também serviram para complementar as espécies de rapinantes da área.

\section{Pontos de amostragem}

a) Ponto $1\left(23^{\circ} 52^{\prime} 01.00^{\prime \prime S} 52^{\circ} 43^{\prime} 31.25^{\prime \prime} \mathrm{O}\right)$ : localizado na região leste da reserva, com vegetação próxima a borda bem conservada, apresentando árvores de grande porte com dossel alto e subosque denso. $\mathrm{O}$ entorno é caracterizado por área de cultivo de trigo.

b) Ponto 2 (2349'20.95"S 5246'54.32”O): localizado na região noroeste da RBP, apresenta vegetação em regeneração, devido a um incêndio a cerca de 15 anos atrás. A área de entorno é caracterizada por plantio de cana-de-açúcar (Saccharum $\mathrm{sp}$ ).

c) Ponto 3 (2352'24.36”S 52॰42’41.18”O): localizado ao leste da RBP, apresenta uma vegetação conservada, com dossel alto e subosque denso. A área de entorno é caracterizada por pastagem abandonada com pequenas árvores e arbustos distribuídos de forma esparsa.

d) Ponto 4 (2351'29.39"S 5247’39.52”O): localizado na região oeste da RBP, a vegetação do local é preservada, com dossel alto e subosque denso, com pastagem na área de entorno.

e) Ponto $5\left(23^{\circ} 53^{\prime} 36.91^{\prime \prime} \mathrm{S} 52^{\circ} 48^{\prime} 07.41^{\prime \prime} \mathrm{O}\right)$ : localizado ao sul da RBP, apresenta árvores altas e

FIGURA 1: Localização dos pontos de amostragem na Reserva Biológica das Perobas, Tuneiras do Oeste, Paraná

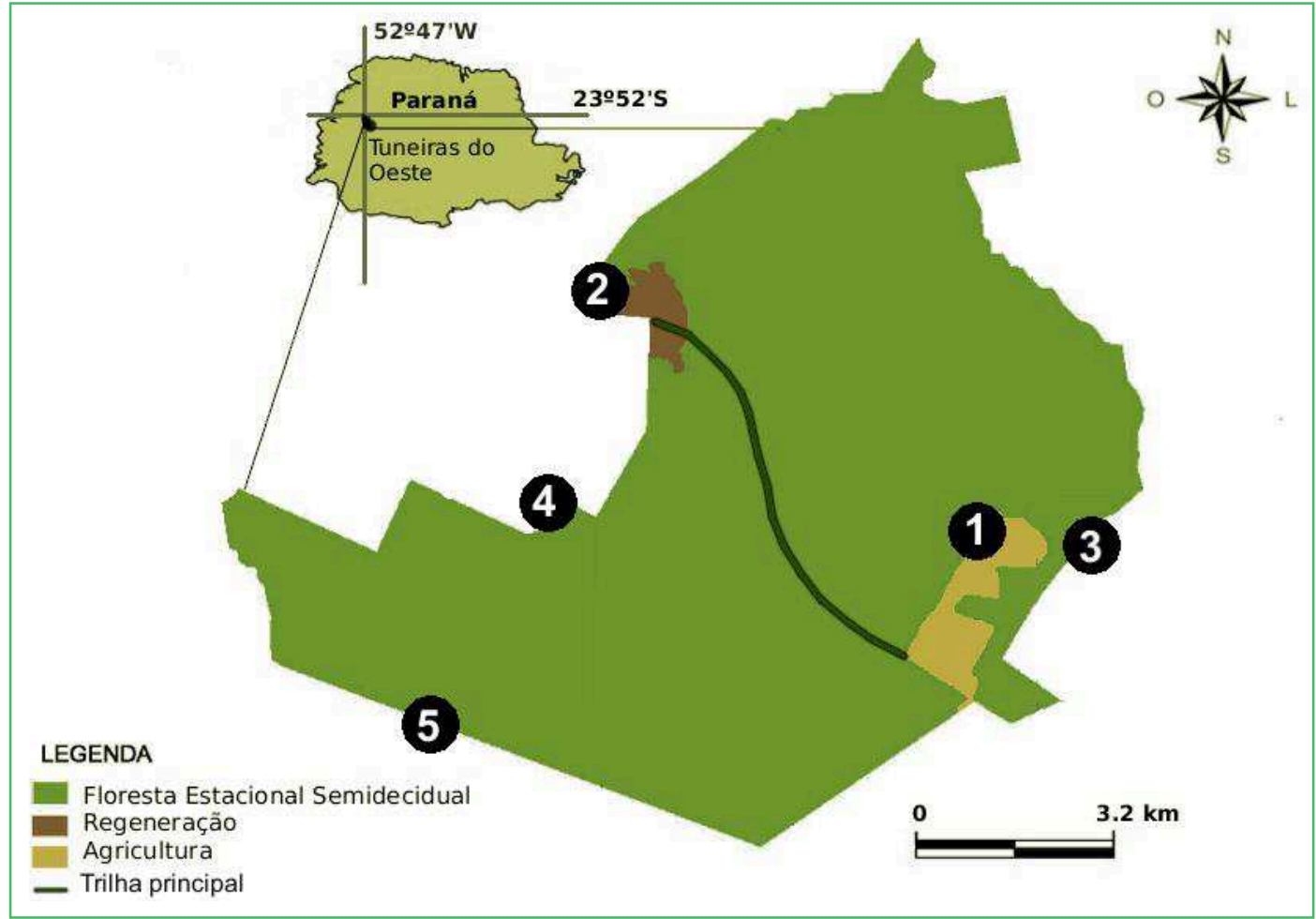


subosque alterado. A floresta está margeada pela estrada boiadeira (BR-427), não pavimentada e com baixo trânsito de veículos. A área de entorno é caracterizada por pastagens.

f) Trilha principal (23050'29.69”S $52^{\circ} 44^{\prime} 54.62$ ”O): localizado na antiga estrada já desativada, que corta a RBP de leste a oeste, com cerca de 2,5 m de largura e extensão de aproximadamente $8,3 \mathrm{~km}$. A trilha apresenta vegetação em diferentes estágios de regeneração predominando áreas mais conservadas, com dossel alto e subosque denso.

Para registrar os rapinantes diurnos, foi utilizado o método de contagem por pontos fixos (FULLER; MOSHER, 1987), o qual consiste no estabelecimento de pontos de observação distribuídos na área de estudo, com amostragens iniciando do amanhecer até as $14 \mathrm{~h}$. Os pontos foram alocados nas bordas da floresta, apresentando condições favoráveis à detecção de aves de rapina, ou seja, com boa visibilidade acima do dossel da mata. Também foram realizados transectos na trilha principal a fim de registrar as espécies típicas do interior da floresta. Para os Strigiformes, foram realizados pontos de escuta e playback, onde foi reproduzido o som das possíveis espécies ocorrentes no Paraná. Em cada ponto, inicialmente, foram realizados três minutos de escuta, seguidos de três minutos de reproduções e terminando com cinco minutos finais de espera, respeitando um provável período de letargia (MOSHER et al., 1990). A técnica foi empregada nas três primeiras horas da noite, horário em que as corujas são mais ativas e detectáveis (GRANZINOLLI; MOTTA-JUNIOR, 2008), com esforço amostral de $20 \mathrm{~h}$. Nos pontos fixos, foram consideradas nas observações apenas as aves registradas dentro de um ângulo de visão de um arco de $120^{\circ}$, com raio de detecção de $200 \mathrm{~m}$ (Figura 2), com esforço amostral de $32 \mathrm{~h}$ por ponto, enquanto que no transecto foram anotadas as espécies com até $100 \mathrm{~m}$ de distância para cada lado do observador, com esforço amostral total de $30 \mathrm{~h}$. O esforço amostral total deste trabalho em campo foi de aproximadamente $210 \mathrm{~h}$. O ordenamento taxonômico bem como os nomes em português seguiram CBRO (2014).

A estimativa da frequência de ocorrência das espécies teve propósito de estipular o índice de abundância na área em estudo. $O$ cálculo foi determinado através do índice de Lindsdale, conforme BUGALHO (1974), no qual se calcula através da divisão do número de vezes que a espécie foi registrada pelo número

FIGURA 2: Representação esquemática pontos de amostragem (A) e transecção (B) para o levantamento das aves de rapina na Reserva Biológica das Perobas, Paraná.

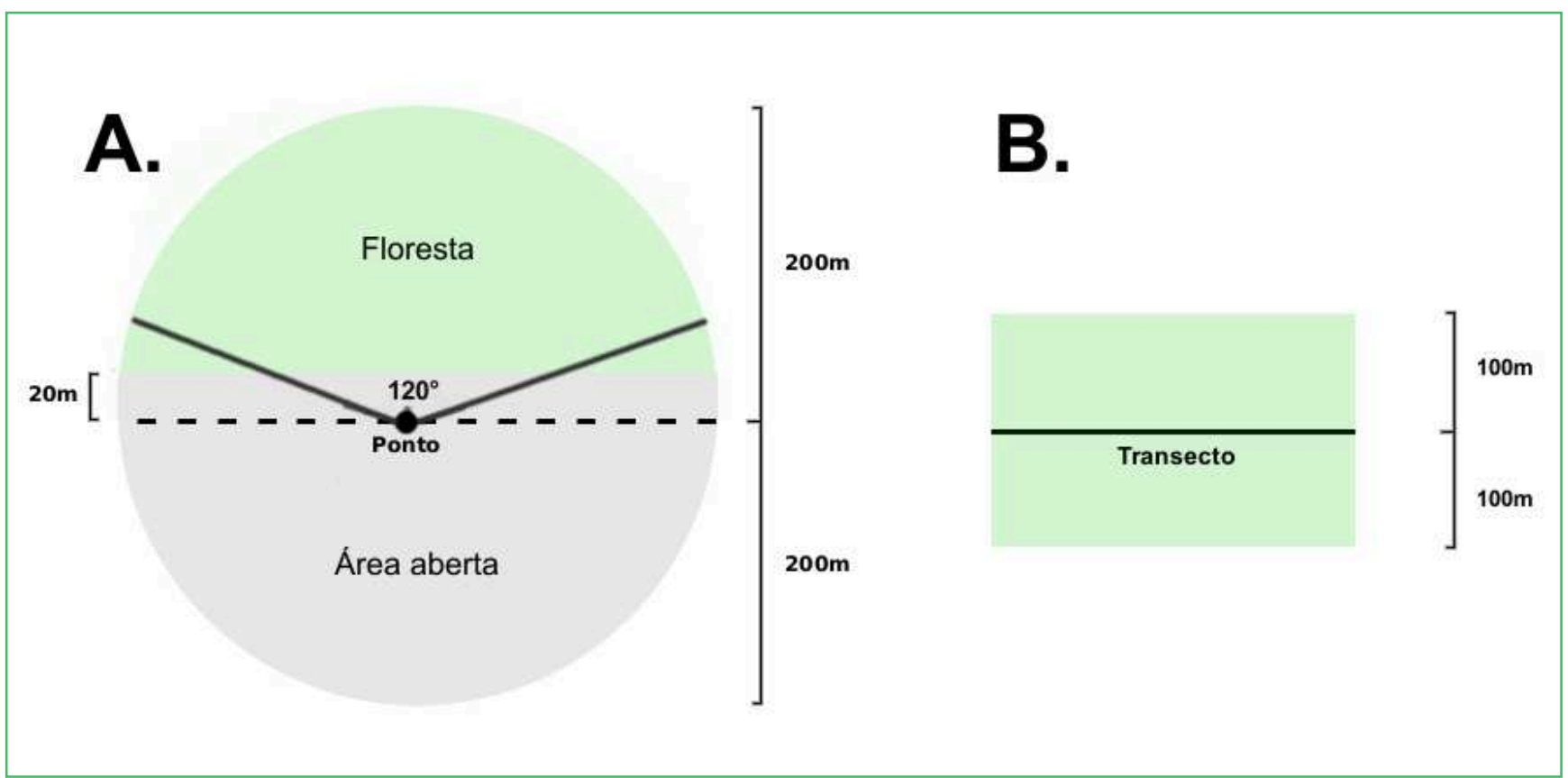


total de amostragens, sendo o resultado expresso em porcentagem. Os índices de abundância foi definido de acordo com AZEVEDO et al. (2003): Muito abundante, espécies registradas entre 81-100\%; abundante, 61$80 \%$; frequente, $41-60 \%$; ocasional, $21-40 \%$ e; rara, $1-20 \%$. Para os Strigiformes, o cálculo da frequência de ocorrência foi realizado considerando apenas as campanhas noturnas.

\section{Resultados}

Foram registradas 25 espécies de aves de rapina na Reserva Biológica das Perobas (Tabela 1). As espécies mais comuns observadas foram Cathartes aura, Rupornis maginostris, Caracara plancus e Megascops choliba, com frequência de ocorrência acima de $95 \%$. A área de entorno, e suas paisagens singulares, foram

TABELA 1: Lista sistemática das aves de rapina registradas na Reserva Biológica das Perobas. (FO) Frequência de ocorrência: (Ma) Muito abundante: espécies registradas entre $81 \%$ e 100\%; (A) Abundante: entre 61-80\%; (Fr) Frequente: 41-60\%; (O) Ocasional: 21-40\%; (Ra) Rara: 1-20\%. Pontos de amostragem: 1, 2, 3, 4, 5, (Tr) Trilha principal, (Fp) Fora do ponto de amostragem. (Am) Ambiente de ocorrência do táxon na RBP: (F) florestal, (A) área aberta. Status (St), onde $r$ = residente; $m$ = migratório. - Bibliografia utilizada (SICK, 1997; CBRO, 2014). Referência: (SE) registro de literatura realizado por SIQUEIRA (1999).

\begin{tabular}{|c|c|c|c|c|}
\hline Táxon & FO & Pontos & Am & ST \\
\hline \multicolumn{5}{|l|}{ Cathartiformes } \\
\hline Cathartes aura (Linnaeus, 1758) & $\mathrm{Ma}$ & $1,2,3,4,5, \operatorname{Tr}$ e Fp & $\mathrm{F}, \mathrm{A}$ & $\mathrm{r}$ \\
\hline Coragyps atratus (Bechstein, 1793) & $\mathrm{Ma}$ & $1,2,3,4,5$ e Fp & $\mathrm{F}, \mathrm{A}$ & $\mathrm{r}$ \\
\hline Sarcoramphus papa (Linnaeus, 1758) & $\mathrm{Ra}$ & 3 & $\mathrm{~F}, \mathrm{~A}$ & $\mathrm{r}$ \\
\hline $\begin{array}{l}\text { Accipitriformes } \\
\text { Accipitridae }\end{array}$ & \multicolumn{3}{|c|}{ Accipitriformes } & \\
\hline Leptodon cayanensis (Latham, 1790) & $\mathrm{Ra}$ & $\operatorname{Tr}$ & $\mathrm{F}$ & $\mathrm{r}$ \\
\hline Elanoides forficatus (Linnaeus, 1758) & $\mathrm{O}$ & 5 & $\mathrm{~F}$ & $\mathrm{~m}$ \\
\hline Circus buffoni (Gmelin, 1788) & $\mathrm{Ra}$ & $\mathrm{Fp}$ & A & $\mathrm{r}$ \\
\hline Ictinia plumbea (Gmelin, 1788) & $\mathrm{O}$ & 2, 4, Tr,e Fp & $\mathrm{F}$ & $\mathrm{m}$ \\
\hline Geranospiza caerulescens (Vieillot, 1817) & $\mathrm{Ra}$ & 5 & $\mathrm{~F}, \mathrm{~A}$ & $\mathrm{r}$ \\
\hline Rupornis magnirostris (Gmelin, 1788) & Ma & $1,2,3,4,5, \operatorname{Tr} \mathrm{e} F \mathrm{p}$ & $\mathrm{F}, \mathrm{A}$ & $\mathrm{r}$ \\
\hline Geranoaetus albicaudatus (Vieillot, 1816) & $\mathrm{O}$ & 1,2 e Fp & A & $\mathrm{r}$ \\
\hline Buteo brachyurus (Vieillot, 1816) & $\mathrm{Ra}$ & $\mathrm{Fp}$ & $\mathrm{F}, \mathrm{A}$ & $\mathrm{r}$ \\
\hline Spizaetus melanoleucus (Vieillot, 1816) & $\mathrm{Ra}$ & 1,5 & $\mathrm{~F}$ & $\mathrm{r}$ \\
\hline \multicolumn{5}{|l|}{ Strigiformes } \\
\hline Megascops choliba (Vieillot, 1817) & Ma & $\operatorname{Tr}$ & $\mathrm{F}$ & $\mathrm{r}$ \\
\hline Megascops atricapilla (Temminck, 1822) & A & $\operatorname{Tr}$ & $\mathrm{F}$ & $\mathrm{r}$ \\
\hline Pulsatrix koeniswaldiana (Bertoni \& Bertoni, 1901) & $\mathrm{Ra}$ & $\operatorname{Tr}$ & $\mathrm{F}$ & $\mathrm{r}$ \\
\hline Strix virgata (Cassin, 1849) & A & $\operatorname{Tr}$ & $\mathrm{F}$ & $\mathrm{r}$ \\
\hline Glaucidium brasilianum (Gmelin, 1788) & A & $\operatorname{Tr}$ & $\mathrm{F}$ & $\mathrm{r}$ \\
\hline Athene cunicularia (Molina, 1782) & $\mathrm{O}$ & 2 e Fp & A & $\mathrm{r}$ \\
\hline Asio flammeus (Pontoppidan, 1763) & $\mathrm{Ra}$ & 2 & A & $\mathrm{r}$ \\
\hline \multicolumn{5}{|l|}{ Falconiformes } \\
\hline Caracara plancus (Miller, 1777) & Ma & $1,2,3,4,5$ e Fp & A & $\mathrm{r}$ \\
\hline Milvago chimachima (Vieillot, 1816) & $\mathrm{Ra}$ & $\mathrm{Fp}$ & A & $\mathrm{r}$ \\
\hline Micrastur semitorquatus (Vieillot, 1817) & $\mathrm{Ra}$ & $5, \operatorname{Tr}$ & $\mathrm{F}$ & $\mathrm{r}$ \\
\hline Falco sparverius (Linnaeus, 1758) & $\mathrm{Fr}$ & 1,3 e Fp & A & $\mathrm{r}$ \\
\hline Falco rufigularis (Daudin, 1800) ${ }^{\mathrm{SE}}$ & - & - & $\mathrm{F}, \mathrm{A}$ & $\mathrm{r}$ \\
\hline Falco femoralis (Temminck, 1822) & $\mathrm{Ra}$ & $1 \mathrm{e} \mathrm{Fp}$ & $\mathrm{A}$ & $\mathrm{r}$ \\
\hline
\end{tabular}


relevantes no aumento da riqueza de espécies, visto que $32 \%$ das espécies foram registradas somente nesses ambientes. Rapinantes com registros escassos ou mal documentados no noroeste do estado também foram registradas, destacando-se os casos de Megascops atricapilla, Asio flammeus, Strix virgata, Pulsatrix koeniswaldiana, Micrastur semitorquatus e Spizaetus melanoleucus (Figura 3).

Durante as amostragens com os pontos fixos, entre junho de 2009 e julho de 2010, foram obtidos 184 contatos com rapinantes diurnos (famílias Accipitridae, Falconidae e Cathartidae), sendo que 55\% foram realizados entre as 9 e $12 \mathrm{~h}$ (Figura 4), demonstrando ser a faixa de horário mais eficiente para avistamento de rapinantes planadores. Após as 13 h, o número de contatos representou apenas $9 \%$ do total obtido. Algumas espécies foram registradas apenas em horários específicos, como Micrastur semitorquatus, amostrada somente entre as 7 e $8 \mathrm{~h}$ no interior da floresta, logo após o amanhecer. A curva, acumulada de todos os métodos, como era de se esperar para as primeiras campanhas não apresentou tendência à estabilização, a partir dos últimos meses, tal tendência ficou mais evidente (Figura 5).

\section{Discussão}

O total de espécies registrado nesse estudo corresponde a $37 \%$ do total de rapinantes encontrados no estado do Paraná (SCHERER-NETO et al., 2011) e 52\% do total conhecido para a região (STRAUBE et al., 1996;

FIGURA 3: Registros relevantes de rapinantes obtidos na Reserva Biológica das Perobas: (A) Spizaetus melanoleucus, avistado dia 27/09/09, voando com presa não-identificada nas garras sobre a estrada boiadeira, entorno da RBP; (B) Leptodon cayanensis, registrado dia 13/09/09, em voo sobre a floresta na RBP; (C) Strix virgata, observada dia 01/11/11, após reprodução de playback no interior da RBP; (D) Asio flammeus, registrada dia 12/09/09, voando em área de pastagem no entorno da RBP. Fotos: Willian Menq.

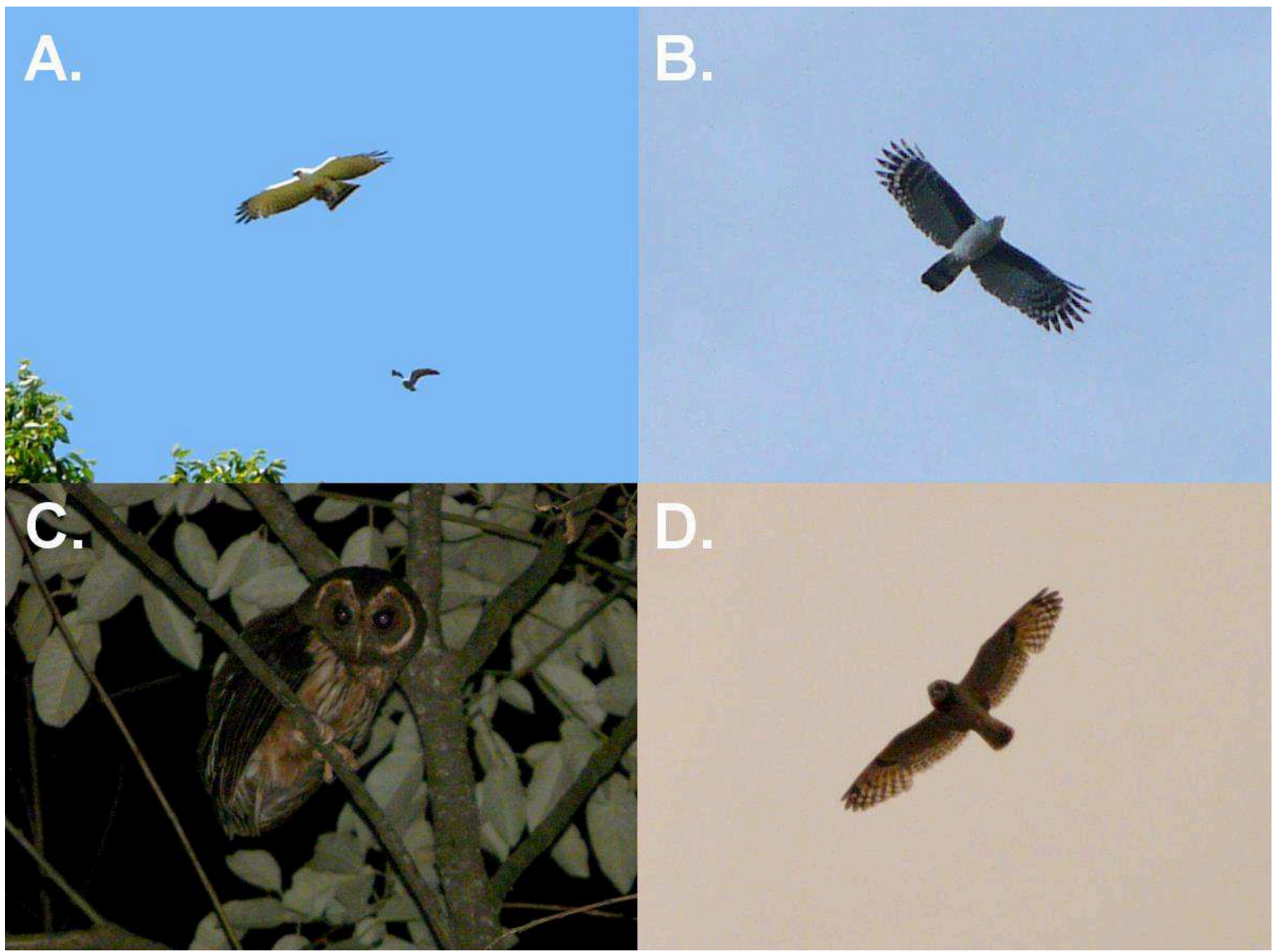


FIGURA 4: Número de contatos com rapinantes diurnos (Accipitridae, Falconidae e Cathartidae) nos diferentes horários, nos pontos amostrais na Reserva Biológica das Perobas, Paraná.

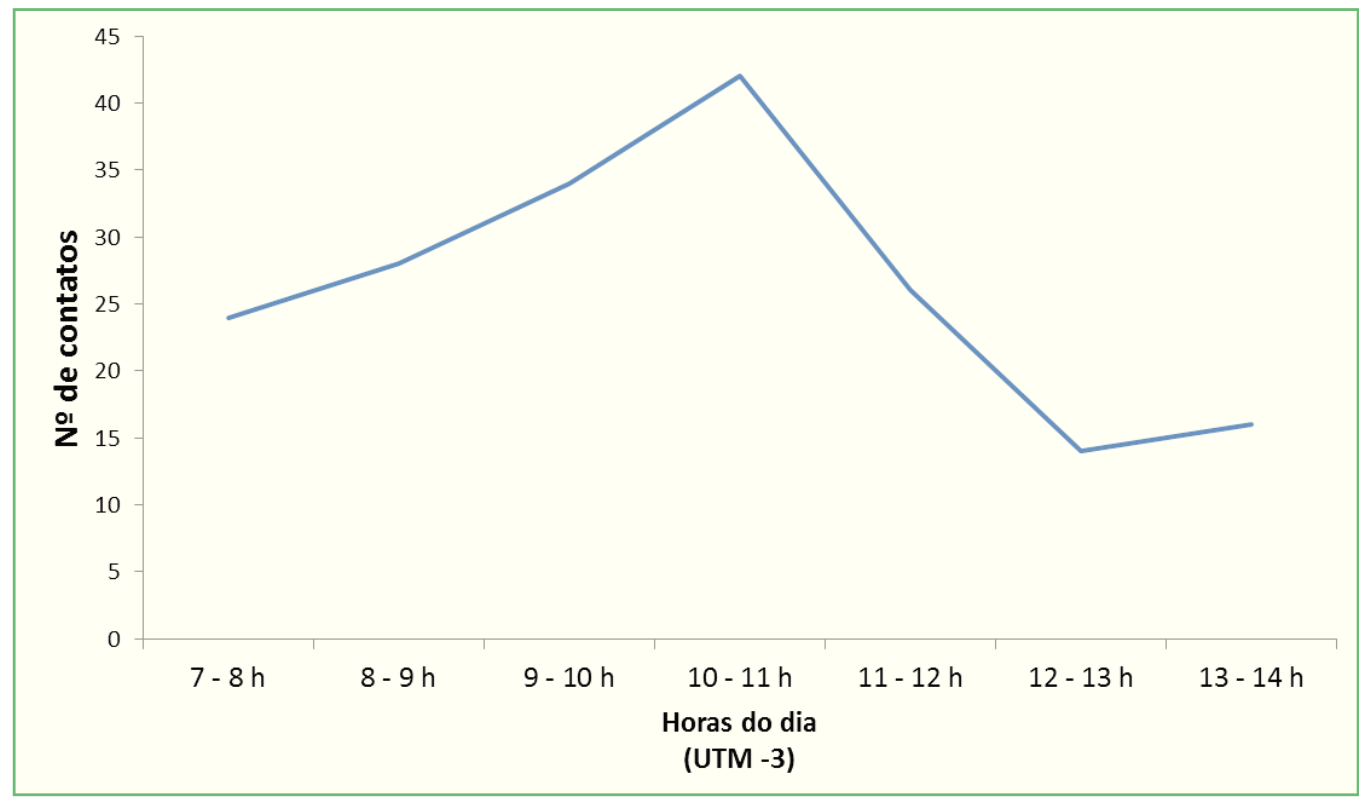

FIGURA 5: Riqueza acumulada durante as amostragens no período de junho de 2009 a janeiro de 2012 na Reserva Biológica das Perobas, Paraná.

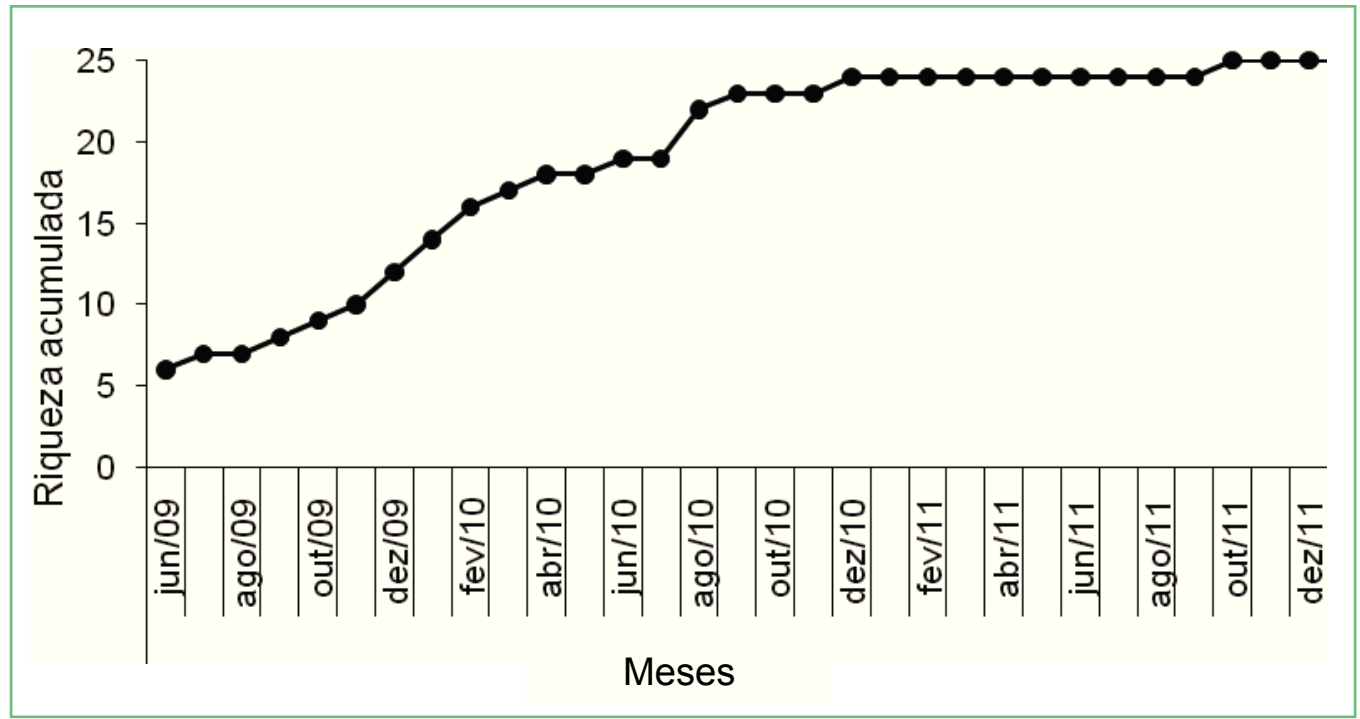

SCHERER-NETO; CARRANO, 1998; BORNSCHEIN; REINERT, 2000; STRAUBE; URBEN-FILHO, 2005; GIMENES et al., 2007; SCHERER-NETO et al., 2008; SCHERER-NETO; BISPO, 2011; LIMA, 2013), o que pode ser considerado como uma alta riqueza na área da RBP.
A RBP abriga diversas espécies estritamente florestais e sensíveis a perturbação antrópica, dentre elas destacam-se M. atricapilla, S. virgata, P. koeniswaldiana, $M$. semitorquatus e $S$. melanoleucus (STOTZ et al., 1996; SICK, 1997), ausentes na maioria dos pequenos fragmentos do noroeste do estado (STRAUBE; URBENFILHO, 2005). Dentre os registros mais significativos, 
destaca-se o de $S$. melanoleucus, observada somente em duas oportunidades na RBP. O primeiro registro foi obtido no dia 13 de junho de 2009 na borda leste da $\mathrm{RBP}$, onde um indivíduo adulto foi registrado pousado no alto de exemplar morto de peroba-rosa (Aspidosperma polyneuron, Apocynaceae). O espécime permaneceu na árvore até ser acuado por um indivíduo de Falco sparverius, voando para o interior da vegetação. No dia 27 de setembro de 2009, outro adulto de S. melanoleucus foi avistado voando próximo à borda sul da RBP com um pequeno mamífero não-identificado entre as garras. O gavião era notadamente menor que o da observação anterior, e devido à diferença de tamanho, é possível supor que se tratava de indivíduos de sexos diferentes, o maior (do primeiro registro) uma fêmea e o outro um macho.

Spizaetus melanoleucus é estritamente florestal, naturalmente rara e está regionalmente ameaçada de extinção em todos os estados que possuem um livro vermelho elaborado, como Rio de Janeiro (ALVES et al., 2000), Espírito Santo (SIMON et al., 2007), Minas Gerais (DRUMMOND et al., 2008), São Paulo (SILVEIRA et al., 2009) e Rio Grande do Sul (RIO GRANDE DO SUL, 2014) e Paraná, onde é categorizado como "em perigo" (STRAUBE et al., 2004). São escassos os registros publicados da espécie em áreas sob domínio da Floresta Estacional Semidecidual no Paraná (SANTOS; COPATTI, 2009), sendo que os mais recentes foram obtidos no município de Foz do Iguaçu (FARIAS, 2015), Capanema (PIERI, 2015) e na Estação Ecológica de Luiziana (SIQUEIRA, 2011). A RBP constitui um dos poucos locais da região capaz de abrigar $S$. melanoleucus, e o baixo número de contatos nesse estudo sugere que a espécie tenha uma baixa abundância e seja de difícil detecção na RBP.

Pouco se sabe sobre a distribuição e ecologia de A. flammeus no sul do Brasil. No Paraná é considerada como "dados insuficientes" (STRAUBE et al., 2004). O comportamento da espécie pode ser um dos fatores que influenciaram em sua detecção, já que ocupa campos naturais, áreas de agricultura e pastagens que não costuma ter o mesmo volume de visitas de pesquisadores se comparado às áreas florestadas (KÖNIG; WEICK, 2008). A área de entorno da RBP é predominada por áreas de cultivo de culturas anuais, pastagens e canaviais, ambiente a qual a espécie costuma habitar. Dentre as florestais, Strix virgata, também encontra-se mal documentada no estado, categorizada como "dados insuficientes" no Paraná, Rio Grande do Sul e Rio de janeiro (ALVES et al., 2000; STRAUBE et al., 2004; RIO GRANDE DO SUL, 2014). Na RBP aparenta ser abundante, registrada diversas vezes na trilha principal no interior da mata $(\mathrm{FO}=80 \%)$. Da mesma forma, observou-se- em relação a $M$. atricapilla, abundante na $\mathrm{RBP}(\mathrm{FO}=75 \%)$ e mal documentada nas áreas sob domínio da Floresta Estacional Semidecidual no estado, demonstrando uma maior raridade da espécie para essa fitofisionomia quando comparada a Florestas Ombrófila Densa.

Elanoides forficatus, sempre registrada entre os meses de agosto e setembro nos municípios da região (W.M., observação pessoal), foi avistada somente no mês de setembro na RBP. É um gavião migratório, que vive em bandos de vários indivíduos e se reproduz no sul do Brasil (SICK, 1997). Porém, nunca foi observado nos meses de outubro a dezembro na região, sugerindo que os registros da RBP, sejam apenas de indivíduos que ainda estão se deslocando para o destino final de invernagem e adotando "stopover", que são paradas de pouso temporário para se alimentar e descansar, antes de chegar à área definitiva de reprodução. Em março de 2013, Fernandes (2013) registrou um bando de quase 150 indivíduos voando em um parque urbano de Maringá, a cerca de $100 \mathrm{~km}$ da RBP. Nos dias seguintes, o bando desapareceu do parque, provavelmente realizando stopover, voltando da migração rumo ao norte do país. Essas informações reforçam a hipótese de permanência na região apenas em paradas temporárias.

Embora a curva de acumulação demostre uma tendência a estabilização, espécies florestais relativamente comuns como Harpagus diodon, Accipiter striatus e Micrastur ruficollis, que contam com registros nos fragmentos da região (STRAUBE; URBEN-FILHO 2005; SCHERER-NETO et al., 2008; BISPO, 2011), não foram observadas neste estudo, sendo provável sua ocorrência na área. Acredita-se que Spizaetus ornatus, rapinante raro e críptico, também ocorra no interior da RBP. É um gavião estritamente 
florestal, de baixas densidades populacionais e de difícil detecção, já que raramente plana acima do dossel da mata e dificilmente vocaliza, como já observado por $\mathrm{D}$. Kajiwara (comunicação pessoal) no interior do Paraná. Conta com poucos registros no estado e recentemente foi registrado na Estação Ecológica Luiziana, fragmento florestal de 1.166 ha, localizado a cerca de $70 \mathrm{~km}$ da reserva (PREFEITURA MUNICIPAL DE LUIZIANA, 2009; SIQUEIRA, 2012).

Em síntese, os dados obtidos neste estudo demonstram que a Reserva Biológica das Perobas apresenta uma rica diversidade de aves de rapina, incluindo a presença de espécies raras. Assim, podemos apontar a UC como uma das mais importantes áreas do norte e noroeste do Paraná para a conservação e manutenção de populações relictuais de rapinantes florestais. Considerando a extensão da RBP, os registros da região e a distribuição geográfica de várias espécies, acredita-se que a riqueza de rapinantes seja ainda maior do que a registrada neste estudo. Nesse sentido, sugerese que novos estudos com o grupo sejam realizados na reserva, com amostragens que contemplem novas áreas da floresta, especialmente o interior da mesma, o que proporcionará o registro de espécies raras ou de hábitos crípticos, como rapinantes dos gêneros Accipiter e Spizaetus e Strigiformes.

Estudos relacionados à biologia e à distribuição das aves de rapina em Unidades de Conservação são de grande importância para promover e direcionar monitoramentos posteriores, gerar conhecimento sobre as espécies, somando subsídios para a elaboração de estratégias de conservação das mesmas.

\section{Agradecimentos}

Agradecemos ao Jean Ferreira Copatti pela ajuda e companhia de campo. Ao ICMBio e a equipe da Reserva Biológica das perobas: Carlos de Giovanni, Antônio Guilherme e Deusdeti pelo convite da pesquisa, pelo apoio e logística de campo. Agradecemos também ao Cesumar pela concessão da bolsa de iniciação científica. Ao Selson Garutti, Jessica Moraes Nascimento e Jorge Albuquerque, pelas críticas e sugestões do manuscrito.

\section{Referências}

AlveS, M. A. S.; PACHECO, J. F.; GONZAGA, L. A. P.; CAVALCANTI, R. B.; RAPOSO, M. A.; YAMASHITA, C.; MACIEL, N. C.; CASTANHEIRA, M. Aves. In: A fauna ameaçada de extinção do estado do Rio de Janeiro. Rio de Janeiro: Universidade do Estado do Rio de Janeiro, 2000. p. 113-124.

ANJOS, L. Consequências biológicas da fragmentação no norte do Paraná. Instituto de Pesquisa e Estudos Florestais, Piracicaba, v. 12, p. 87-94, 1998.

ANJOS, L.; MENQ, W. Aves. In: MAGALHÃES-JUNIOR, C. A. O.; ORTÊNCIO-FILHO, H.; DELARIVA, R. L. (Ed.). Reserva Biológica das Perobas - uma ilha de biodiversidade no noroeste paranaense. Curitiba: Instituto Tecnológico de Transportes e Infraestrutura, 2014. p. 32-39.

AZEVEDO, M. A. G.; MACHADO, D. A.; ALBUQUERQUE, J. L. B. Aves de rapina na Ilha de Santa Catarina, SC: composição, frequência de ocorrência, uso de habitat e conservação. Revista Brasileira de Ornitologia, Belém, v. 1, n. 4, p. 75-81, 2003.

BORNSCHEIN, M. R.; REINERT, B. L. Aves de três remanescentes florestais do norte do estado do Paraná, sul do Brasil, com sugestões para a conservação e manejo. Revista Brasileira de Zoologia, Curitiba, v. 17, n. 3, p. 615-636, 2000.

BUGALHO, J. F. Métodos de recenseamento de aves. Lisboa: Monumental LDA, 1974. 108 p.

CAMPOS, J. B. Desmatamentos no Paraná. Cadernos de Biodiversidade, Curitiba, v. 1, n. 1, p. 1-2, 1998.

CBRO - COMITE BRASILEIRO DE REGISTROS ORNITOLÓGICOS. Listas das aves do Brasil. 2014. 11 ed. Disponível em: <http://www.cbro.org.br>. Acesso em: 10 dez. 2014. DRUMMOND, G.; MACHADO, A. B. M.; MARTINS, C. S.; MENDONÇA, M. P.; STEHANN, J. P. Listas das espécies da flora e da fauna ameaçadas de extinção do estado de Minas Gerais. Belo Horizonte: Fundação Biodiversitas, 2008. CD-ROM. FARIAS, C. [WA1629527, Spizaetus melanoleucus (Vieillot, 1816)]. 2015. Wiki Aves. Disponível em: <http://www.wikiaves. com/1629527>. Acesso em: 30 mar. 2015.

FERGUSON-LEES, J.; CHRISTIE, D. A. Raptors of the world. Boston: Houghton Miffling Company, 2001. 992 p.

FERNANDES, E. [WA906820, Elanoides forficatus (Linnaeus, 1758)]. 2013. Wiki Aves. Disponível em: <http://www.wikiaves. com/906820>. Acesso em: 11 maio 2015.

FULLER, M.; MOSHER, J. A. Raptor survey techniques. In: BIRD, D. M.; BILDSTEIN, K. L.; BARBER, D. R.; ZIMMERMAN, A. (Ed.). Raptor management techniques manual. Washington: National Wildlife Federation, 1987. p. 37-66.

GIMENES, M. R.; LOPES, E. V.; LOURES-RIBEIRO, A.; MENDONÇA, L. B.; ANJOS, L. Aves da planície alagável do alto Rio Paraná. Maringá: Universidade Estadual de Maringá, 2007. 281 p.

GRANZINOLLI, M. A. M.; MOTTA-JUNIOR, J. C. Aves de rapina: levantamento, seleção de habitat e dieta. In: MATTER, S. V.; STRAUBE, F. C.; ACCORDI, I.; PIACENTINI, V.; CÂNDIDO JR, J. F. (Org.). Ornitologia e conservação: ciência aplicada, técnicas de pesquisa e levantamento. Rio de Janeiro, Technical Books. 2008. p. 169-187. 
ICMBIO - INSTITUTO CHICO MENDES DE CONSERVAÇÃO DA BIODIVERSIDADE. Plano de manejo da Reserva Biológica das perobas. Brasília: ICMBIO, 2012. 198 p.

KÖNIG, C.; WEICK, F. Owls of the world. New Haven: Yale University Press, 2008. 519 p.

LIMA, B. [WA1113218, Buteo albonotatus Kaup, 1847]. 2013. Wiki Aves. Disponível em: <http://www.wikiaves.com/1113218> Acesso em: 8 jul. 2015.

LOURES-RIBEIRO, A.; ANJOS, L. Falconiformes assemblages in a fragmented landscape of the Atlantic Forest in southern Brazil. Brazilian Archives of Biology and Technology, Curitiba, v. 49, n. 1, p. 149-162, 2006.

MOSHER, J. A.; FULLER, M. R.; KOPENY, M. Surveying woodland raptors by broadcst of conspecific vocalization. Journal Field Ornithology, Houston, v. 61, n.4, p. 453-461, 1990.

OLMOS, F.; PACHECO, J. F.; SILVEIRA, L. F. Notas sobre aves de rapina (Cathartidae, Acciptridae e Falconidae) brasileiras. Revista Brasileira de Ornitologia, Belém, v. 14, n. 4, p. 401-404, 2006.

PEEL, M. C.; FINLAYSON, B. L.; MCMAHON, T. A. Updated world map of the Köppen-Geiger climate classification. Hydrology and Earth System Sciences, Delft, v. 11, p. 1633-1644, 2007.

PIERI, A. [WA1698873, Spizaetus melanoleucus (Vieillot, 1816)]. 2015. Wiki Aves. Disponível em: <http://www.wikiaves.com/ 1698873 >. Acesso em: 25 set. 2015.

PREFEITURA MUNICIPAL DE LUIZIANA. Decreto no 441 de 23 de março de 2009. Cria a Estação de Unidade de Conservação Ecológica Municipal em área rural de Proteção Integral, e dá outras providências. 2009.

RIO GRANDE DO SUL. Táxons da fauna silvestre do estado Rio Grande do Sul ameaçadas de extinção. Porto Alegre: Estado do Rio Grande do Sul (Decreto no 51.797 , de 8 de setembro de 2014)

SANTOS, R. E. F.; SCHERER-NETO, P.; ALBUQUERQUE, J. L. B. Gaviões. In: INSTITUTO AMBIENTAL DO PARANÁ (Ed.). Planos de conservação para espécies de aves ameaçadas no Paraná. Curitiba: IAP/Projeto Paraná Biodiversidade, 2009. p. 169-197.

SANTOS, W. M.; COPATTI, J. F. Registro documentado de Spizaetus melanoleucus (Falconiformes: Accipitridae) na Reserva Biológica das Perobas, estado do Paraná. Atualidades Ornitológicas, Ivaiporã, v. 151, p. 18-19, 2009.

SCHERER-NETO, P.; BISPO, A. A. Avifauna do Parque Estadual de Vila Rica do Espírito Santo, Fênix, Paraná. Biota Neotropica, Campinas, v. 11, p. 317-329, 2011.

SCHERER-NETO, P.; CARRANO, E. Gampsonyx swainsonii (Vigors, 1825) no estado do Paraná. Atualidades Ornitológicas, Ivaiporã, v. 82, p. 2, 1998.
SCHERER-NETO, P.; CARRANO E.; RIBAS F. C. Composição e conservação da avifauna da Estação Ecológica do Caiuá, noroeste do Paraná e regiões adjacentes. Cadernos de Biodiversidade, Curitiba, v. 6, n. 1, p. 32-45, 2008.

SCHERER-NETO, P.; STRAUBE, F. C.; CARRANO, E.; URBENFILHO, A. Aves do Paraná: história, lista anotada e bibliografia. Curitiba: Hori Cadernos Técnicos, 2011. 130 p.

SICK, H. Ornitologia brasileira. Rio de Janeiro: Nova Fronteira, 1997. $912 \mathrm{p}$

SILVEIRA, L. F.; BENEDICTO, G. A.; SCHUNCK, F.; SUGIEDA, A. M. Aves. In: BRESSAN, P. M.; KIERULFF, M. C.; SUGIEDA, A. M. (Org.). Fauna ameaçada de extinção no estado de São Paulo: Vertebrados. São Paulo: Fundação Parque Zoológico de São Paulo e Secretaria do Meio Ambiente, 2009. p. 87-283.

SIMON, J.; ANTAS, P. T. Z.; PACHECO, J. F.; EFE, M.; RIBON, R.; RAPOSO, M. A.; LAPS, R.; MUSSO, C.; PASSAMANI, J. ; PACCAGNELA, S. G. As aves ameaçadas de extinção no estado do Espírito Santo. In: MENDES, S. L.; PASSAMANI, M. (Org.). Livro vermelho das espécies da fauna ameaçada de extinção no estado do Espírito Santo. Vitória: IPEMA, 2007. p. 47-64.

SIQUEIRA, E. L. [WA1323827, Falco rufigularis Daudin, 1800]. 1999. Wiki Aves. Disponível em: <http://www.wikiaves. com/1323827>. Acesso em: 7 abr. 2015.

SIQUEIRA, E. L. [WA419675, Spizaetus melanoleucus (Vieillot, 1816)]. 2011. Wiki Aves. Disponível em: $<$ http://www.wikiaves. com/419675>. Acesso em: 7 abr. 2015.

SIQUEIRA, E. L. [WA667547, Spizaetus ornatus (Daudin, 1800)]. 2012. Wiki Aves. Disponível em: <http://www.wikiaves. com/667547>. Acesso em: 7 abr. 2015.

STOTZ, D. F.; FITZPATRICK, J. W.; PARKER, T. A.; MOSKOVITS, D. K. Neotropical Birds: ecology and conservation. Chicago: University of Chicago Press, 1996. 478 p.

STRAUBE, F. C.; BORNSCHEIN, M. R.; SCHERER-NETO, P. Coletânea da avifauna da região noroeste do estado do Paraná e áreas limítrofes (Brasil). Arquivos de Biologia e Tecnologia, Curitiba, v. 39, p. 193-214, 1996

STRAUBE, F. C.; URBEN-FILHO, A. Observações sobre a avifauna de pequenos remanescentes florestais na região noroeste do Paraná (Brasil). Atualidades Ornitológicas, Ivaiporã, v. 123, p. 10 (resumo), 2005. Disponível em: <http://www.ao.com.br/ download/noroeste.pdf $>$. Acesso em: 7 abr. 2015.

STRAUBE, F. C.; URBEN-FILHO, A.; KAJIWARA, D. Aves. In: MIKICH, S. B.; BERNILS, R. S. (Org.). Livro vermelho da fauna ameaçada no estado do Paraná. Curitiba: Instituto Ambiental do Paraná, 2004. p. 145-496.

THIOLLAY, J. M. Area requirements for the conservation of rainforest and game birds in French Guiana. Conservation Biology, San Francisco, v. 3, p. 128-137, 1989. 